\title{
DIRITTO E TECNOLOGIA
}

\author{
VALERIO ONIDA (*)
}

\begin{abstract}
SuNTO. - L'intervento muove dal rilievo per cui la tecnologia è uno strumento per la trasformazione della realtà di per sé "neutrale", cioè impiegabile per fini diversi e anche opposti, mentre il diritto è uno strumento per orientare, condizionare e governare comportamenti umani in relazione a fini sociali, cioè a ciò che si ritiene giusto o migliore per la società. Di qui l'intrinseco "finalismo" del diritto, e la differenza fondamentale fra il "potere" della tecnica e il potere giuridico che si esercita per i fini sociali e per dirimere conflitti di interessi fra individui e fra collettività. Si esaminano poi le potenzialità e i limiti dell'impiego delle tecniche informatiche per compiere atti giuridici; il nuovo ruolo del diritto di fronte alla crescita di "poteri" tecnologici di raccolta e di utilizzo dei dati; la richiesta al diritto di adeguare la regolazione dei rapporti fra individui (come i rapporti di lavoro) di fronte alle trasformazioni tecnologiche della realtà; i problemi della "delocalizzazione" del diritto legati allo sviluppo di Internet; le nuove richieste al diritto di regolare fenomeni come la manipolazione genetica dell'essere umano e l'impiego della intelligenza artificiale, nell'ottica della salvaguardia dell'essenza dell' "umano".
\end{abstract}

$* * *$

ABSTRACT. - The intervention starts from the observation that technology is a tool for the transformation of reality that is itself "neutral", i.e. usable for different and even opposite purposes, while law is a tool to orient, to condition and to govern human behaviours in relation to social ends, that is to say, what is right or better for society. Hence the intrinsic "finalism" of law, and the fundamental difference between the "power" of technology and the juridical power that is exercised for social purposes and to settle conflicts of interests between individuals and between communities. It then examines the potential and limitations of the use of computer techniques to perform legal acts; the new role of law in the face of the growth of technological "powers" of data collection and use; the demand to adapt the regulation of relationships between individuals (such as labour relationships) in the face of technological changes in reality; the problems of "relocation" of the law related to the development of the Internet; the new demands to legal powers to regulate phenomena such as the genetic manipulation of the human being and the use of artificial intelligence, with a view to safeguarding the essence of being "human".

(*) Professore emerito, Università degli Studi di Milano, Italia.

E-mail: valerio.onida@onidalex.it 
Non essendo, a differenza di chi mi ha preceduto, esperto dei temi specifici trattati, cioè delle nuove frontiere del progresso tecnologico, mi limito qui a qualche riflessione di ordine generale sui rapporti fra scienza, tecnologia e diritto.

\section{UN DIVERSO ORIZZONTE}

L'orizzonte della scienza è il progresso della conoscenza; essa non ha necessariamente altri fini specifici. La tecnologia è uno strumento: avvalendosi delle conoscenze scientifiche, tende a realizzare o a rendere realizzabili delle modifiche della realtà, che possono avere i fini più diversi. Ad esempio un processo tecnico di fissione nucleare può essere impiegato per produrre energia al servizio della società, o per realizzare una bomba capace di distruggere e uccidere.

Il diritto invece tende non a conoscere o a modificare direttamente la realtà, ma a determinare l'orientamento di comportamenti umani per perseguire fini sociali. E' dunque essenzialmente caratterizzato dai fini che persegue. Creare diritto significa porre regole o comandi per i comportamenti umani, regole che impongono, vietano o permettono determinate categorie di comportamenti in vista dei fini che si intendono perseguire. Applicare il diritto significa valutare comportamenti umani concreti alla luce di quelle regole e trarne delle conseguenze a loro volta su altri comportamenti umani, in termini di garanzia di libertà o di risposta a bisogni e a richieste, o in termini di applicazione di sanzioni per l'avvenuta trasgressione di divieti o inosservanza di obblighi, sempre per il perseguimento dei fini scelti.

\section{Potere teCnico E POTERE GIURIDiCO}

L'orizzonte della scienza e della tecnica è l'essere, quello del diritto è il dover essere, l'orizzonte dei fini. Noi parliamo (ambiguità delle parole) di leggi sia per quelle fisiche sia per atti che esprimono e pongono diritto, ma si tratta di due mondi diversi: le prime descrivono una realtà, le seconde prescrivono, cioè tendono, attraverso il condizionamento delle volontà umane, a determinare comportamenti in vista di ciò che si ritiene giusto o migliore per la società. Comportamenti, per lo più, di rilevanza non unicamente individuale, ma sociale. Tra i fini del 
diritto ci può essere la "ricerca della felicità" degli individui, secondo la nota espressione della Dichiarazione di indipendenza degli Stati Uniti, ma solo nel senso di determinare comportamenti umani di rilevanza sociale che possano indirettamente servire agli individui che cercano la felicità, consentendo loro determinate condotte o imponendo o vietando comportamenti, propri o altrui. Per il diritto di solito non rileva ciò che un singolo individuo semplicemente pensa o sogna, ma solo ciò che fa o non fa, dice o non dice (anche se non mancano esempi di tentativi di condizionamento "tecnico" del pensiero del singolo individuo: ma è questo appunto uno dei confini che nella nostra civiltà giuridica riteniamo non debba essere valicato dal "potere", della forza e del diritto).

La tecnologia ci dice che cosa è "tecnicamente" possibile fare o ottenere per modificare la realtà nelle più diverse direzioni; il diritto ci dice che cosa si può o si deve o non si deve fare per perseguire i fini sociali cui si tende.

Anche la nozione di "potere" è diversa. Il "potere" della tecnica è la possibilità di ottenere, attraverso l'applicazione delle conoscenze scientifiche e l'uso degli strumenti, modificazioni della realtà fisica e anche della realtà umana e sociale (in questo senso si dice che la conoscenza è potere), a prescindere dai fini concreti per i quali tali modificazioni si perseguono o si ottengono. Il "potere" giuridico è la possibilità che in una determinata società uno o più individui cerchino o ottengano di emettere "comandi" giuridici (leggi, provvedimenti amministrativi, sentenze, ecc.) diretti ed idonei a determinare comportamenti umani di rilevanza sociale. La tecnologia ci dice che cosa è possibile fare, il diritto che cosa è lecito, o è doveroso, o è vietato fare.

Certo anche il diritto utilizza per i propri fini delle "tecniche" e degli strumenti tecnici: per esempio per rendere conoscibili ai destinatari le regole poste, o per determinare i modi in cui gli individui possono attivare procedimenti giuridici, o per rendere possibili o più facili comportamenti imposti o desiderabili.

Per fare degli esempi, il progressivo uso dell'informatica nei procedimenti giuridici e nei processi è un modo in cui il diritto si avvale di tecniche per i suoi fini; le nuove possibilità tecniche per la produzione di oggetti o per il trattamento dei rifiuti sono materia di prescrizioni da parte del diritto (si pensi all'uso di certe sostanze o ai requisiti imposti per determinati prodotti industriali o agricoli) in vista del fine di consentire o facilitare il conseguimento di obiettivi ritenuti socialmente desiderabili. Il diritto può avvalersi della tecnologia per imporre con- 
dotte tecnicamente possibili e utili per i fini prescelti, come può considerare contrari ai propri fini certi utilizzi magari della stessa tecnologia. Può promuovere o invece vietare ed ostacolare le trasformazioni della realtà rese possibili dalla tecnologia. Lo sviluppo o il più ampio ricorso a nuove tecniche, se non regolato dal diritto, può produrre anche effetti incompatibili con i fini sociali che orientano il diritto. Non tutto quanto infatti è tecnicamente possibile è socialmente desiderabile, e merita dunque di essere consentito o promosso dal diritto.

\section{NeutralitÀ DELLA TECNICA, FINALISMO DEL DIRITTO}

Insomma, la tecnica è "neutra", e può essere impiegata per fini diversi; il diritto è orientato a fini scelti in base a volontarie determinazioni umane, siano esse espresse in "comandi" d'autorità o emergano dalla stessa costante osservanza di regole (la consuetudine).

Ancora, il diritto può operare e opera bilanciamenti quantitativi o qualitativi fra diversi obiettivi tecnicamente perseguibili, tutti desiderabili, ma che si presentano di fatto come reciprocamente del tutto o in parte non compatibili, o meritevoli di limitazioni o di composizione. Così lo sviluppo incontrollato o eccessivo di determinati processi tecnologici di per sé utili può divenire oggetto di limitazioni o di divieti sulla base di regole di diritto, per raggiungere l'equilibrio desiderabile, o l'equilibrio possibile, fra fini non interamente e incondizionatamente compatibili fra loro: si pensi ad esempio ad applicazioni tecnologiche o all'utilizzo massiccio di tecniche di produzione o di uso di beni suscettibili di produrre effetti dannosi o pericolosi sullo stato dell'ambiente e sul clima.

La tecnologia progredisce velocemente, rendendo possibile operare trasformazioni della realtà prima impensabili o impossibili. Il diritto deve dunque adeguare le proprie prescrizioni utilizzando per i propri fini le nuove possibilità, o impedendo o ostacolando l'uso delle nuove tecniche per fini contrastanti con quelli sociali prescelti.

\section{IL DIRITTO E I CONFLITTI DI INTERESSI}

Ancor più delicato si presenta il problema dell'equilibrio fra i fini perseguiti e perseguibili da diversi individui e fra diversi gruppi umani 
o comunità umane, che possono presentarsi come contrastanti fra di loro. Il diritto opera per comporre, prevenire o dirimere i conflitti di interessi, in base a fini che travalicano la sfera dei rispettivi portatori.

Ciò che vale nei rapporti fra individui, può valere nei rapporti fra diverse comunità. Ad esempio l'uso massiccio di determinate tecniche di sfruttamento del terreno può nuocere all'interesse particolare di una comunità e giovare all'interesse di altre. Da sempre nella storia si manifestano i conflitti di interessi tra gruppi, come le comunità insediate in determinati territori. Se la soluzione del conflitto è lasciata al potere della tecnologia, civile o più spesso militare, disponibile per ciascuna comunità, si ottiene quel risultato che fino a pochi decenni fa sembrava l'unico di fatto perseguibile: la soluzione dei conflitti fra Stati "sovrani”, superiorem non recognoscentes, era in definitiva, e anche in linea di diritto, rimessa, quando accordi contrattuali non erano raggiunti o venivano disattesi, alla forza, cioè alla guerra, in cui la superiorità tecnologica di alcuni nei confronti di altri determinava quasi sempre l'esito del conflitto.

E' solo nell'ultimo secolo che si è manifestata per la prima volta l'idea che anche questi tipi di conflitti debbano trovare soluzione nel diritto, che, orientando le scelte, cerchi di realizzare una composizione di interessi fondata sul riconoscimento di fini universali e di interessi comuni (i diritti umani universali, "la pace e la giustizia fra le nazioni" di cui parla l'articolo 11 della nostra Costituzione).

\section{L'INFORMATICA AL SERVIZIO DEL DIRITTO: GIUDICI ROBOT?}

Oggi si pone spesso il tema dell'uso delle tecniche informatiche per compiere atti giuridici: talora si ipotizza addirittura che una decisione giudiziaria, chiamata ad applicare una norma generale ad un determinato caso o rapporto concreto, possa essere adottata non più attraverso la determinazione volontaria di uno o più esseri umani (i giudici), ma da un apparato tecnico che utilizza l'informatica (i c.d. robot giudici). Dietro questa ipotesi sta la crescita esponenziale della possibilità tecnica di accumulare una quantità enorme di dati conoscitivi riguardanti una determinata realtà, e così anche un determinato caso e individuo.

Per esempio si è ipotizzato negli Stati Uniti di affidare ad un programma informatico la determinazione della quantità di pena da infliggere ad un determinato individuo colpevole di un determinato reato, 
per il quale la legge (come di norma accade) stabilisce un minimo e un massimo (c.d. "forbice edittale") entro i cui limiti spetta al giudice determinare in concreto la pena da infliggere, in base a criteri generali che possono attenere ad una valutazione della gravità del fatto o a caratteristiche soggettive del reo (ad esempio una valutazione della sua capacità o propensione a delinquere): è la cosiddetta "dosimetria sanzionatoria". Ora, si può ammettere che sia un programma informatico a fare questa scelta, applicando un algoritmo prodotto sulla base dell'elaborazione di una serie di informazioni sul reo (età, sesso, precedenti, gruppo sociale di appartenenza, ecc.) e di dati su casi analoghi?

Certamente un programma informatico è in grado di elaborare un risultato tenendo conto di una quantità enorme di dati e di criteri, apparentemente meglio e più in fretta di un giudice in carne ed ossa. Ma chi ha scelto e proporzionato l'impiego dei criteri di quantificazione che, ad esempio, riguardano la "propensione a delinquere" di una persona? Se per esempio la statistica (tradotta in un algoritmo) dice che un certo reato è stato commesso in passato con una frequenza maggiore da parte di persone di una certa età o di uno o altro sesso o magari appartenenti a una certa comunità o a una certa etnia, è ammissibile che questo valga come criterio per decidere la pena da assegnare ad un determinato reo? Conta evidentemente la mente umana che ha configurato l'algoritmo; senza dire che siffatti criteri statistici possono essere in contrasto col principio giuridico di non discriminazione sulla base di fattori come il sesso, l'età o l'etnia di appartenenza. In ogni caso la decisione sanzionatoria non può che essere opera di esseri umani cui spetta la responsabilità di decidere, in base al principio di individualizzazione della pena. La conoscenza e l'elaborazione dei dati possono assistere, ma non sostituire l'uomo.

L'atto del giudicare, del resto, non può mai ridursi ad una semplice deduzione logica nella forma di un sillogismo, nel quale la premessa maggiore (la norma) sia interamente data, la premessa minore (il fatto) sia a sua volta "standardizzato", e dunque la conseguenza sia determinata a priori. Anche senza considerare l'infinita variabilità del "fatto", trattandosi di fatti umani, la stessa norma necessita sempre di essere "interpretata", alla luce di altre norme e di molteplici circostanze storiche e valutative. E' ben noto come l'assunto o il mito del giudice semplice "bocca della legge" sia smentito dalla realtà. Tanto più quanto più il sistema normativo (la legge) si arricchisce e si complica, fornendo, certo, criteri oggettivi per la decisione concreta, ma mai potendosi 
ridurre la decisione ad una semplice operazione matematica, affidabile ad uno strumento tecnico, poiché si tratta di "fare giustizia" rispetto a fatti umani concreti, e questo non è un dato, ma piuttosto un fine che può e deve essere perseguito dalla mente e dalla volontà degli uomini, non sostituibili in questo da nessuna "intelligenza artificiale".

A meno di ritenere che anche le determinazioni umane volontarie dipendano interamente e in modo necessitato e inderogabile da "algoritmi" composti da "dati" e quindi interamente dipendenti dai dati "oggettivi" sulla cui base questi algoritmi sono composti: sarebbe una concezione interamente "deterministica" dell'agire umano, che negherebbe nella sua stessa essenza la libertà dell'uomo (ciascuno sarebbe e farebbe esclusivamente e necessariamente ciò che è interamente programmato a priori dall' "algoritmo" che lo condiziona).

\section{IL POTERE DI RACCOLTA ED ELABORAZIONE DI DATI E IL RUOLO DEL DIRITTO}

Questa impostazione potrebbe d'altro lato condurre a ritenere che le determinazioni volontarie degli esseri umani possano, con l'applicazione della tecnologia, essere interamente "programmate", eliminando ogni incognita frutto di libertà, da chi sia in grado di comporre ed imporre l'algoritmo che "determina" l'agire. Il potere dei soggetti che immagazzinano ed elaborano sempre più "dati" potrebbe tradursi in un potere assoluto (molto superiore a quello di ogni "dittatura" storica) di determinazione dell'agire di altri soggetti.

Che oggi si prospettino rischi concreti in questa direzione pare indubbio, di fronte a pratiche ed episodi, ancora limitati, ma potenzialmente generalizzabili, tendenti a influire sulle scelte di numeri elevatissimi di individui, attraverso l'uso delle "banche dati" di cui l'informatica fornisce la disponibilità, la "profilatura" dei singoli individui raggiunti (potenzialmente tutti o quasi), e l'invio sistematico ad essi di "messaggi" automatici tendenti a realizzare forme di "persuasione occulta". La tecnologia, che consente di collegare in rete miliardi di persone, e di "governare" attraverso appositi algoritmi i messaggi inviati e da inviare, apre strade di questo genere. Chi di noi, che usa quotidianamente gli strumenti della "rete", non ha constatato, per esempio dopo aver effettuato un acquisto online, di essere destinatario "automatico" di determinati messaggi pubblicitari o inviti all'acquisto di altri 
prodotti, "mirati" sulla base dei dati raccolti attraverso il nostro accesso alla rete?

Di fronte a questi fenomeni qual è il ruolo del diritto? E' quello di "governare" l'uso della tecnologia usata per influire sull'agire altrui. D'altra parte non è da oggi che le tecniche di persuasione realizzate con le forme della pubblicità commerciale sono oggetto di statuizioni legali, tendenti ad esempio ad imporre la conoscibilità della fonte dei messaggi, ad evitare la formazione di monopoli od oligopoli informativi, a vietare o limitare la diffusione di certi tipi di messaggi. Tanto più questo e altri tipi di regole giuridiche dovranno essere impiegati di fronte al moltiplicarsi degli strumenti tecnici che rendono anonimi e accessibili a tutti i messaggi in rete (oggi si possono fare con semplice "click" sul computer scelte, rese immediatamente conoscibili a un universo sempre più numeroso di utenti, che un tempo avrebbero comportato assai più riflessione e confronti), e alla "delocalizzazione" della fonte dei messaggi.

Oggi il "potere" sociale tende a collocarsi, più che nelle sedi dove tradizionalmente risiedono la "forza" fisica (le armi) o la "forza" economica (il denaro e le risorse materiali) o la "forza" culturale (il pensiero e la sua diffusione), in sedi nuove dove si accumulano una enorme e crescente quantità di "dati" e una crescente capacità tecnica di elaborazione dei medesimi e di diffusione di messaggi. Il ruolo proprio del diritto, di controllare l'uso della forza e di orientare i comportamenti sociali verso obiettivi utili ad una società che voglia essere interamente "umana", deve oggi fare i conti con questa nuova realtà, e affrontare (e speriamo vincere) la "scommessa" di tenere sotto controllo l'uso di questo nuovo potere.

\section{EVOlUZIONE DELla TECNOLOGIA E NUOVE ESIGENZE DI REGO- LAZIONE GIURIDICA DEI RAPPORTI FRA GLI ESSERI UMANI}

Più in generale, oggi la sfida per il diritto è quella di far fronte a fenomeni e aspetti, resi possibili dalle nuove tecnologie, che rendono più difficile e meno utile l'impiego delle "tecniche" normative tradizionali, o che aprono nuovi rischi rispetto ai quali le regole e gli strumenti giuridici esistenti appaiono inadeguati o insufficienti.

Vi sono rapporti sociali - pensiamo a quelli che nella nostra tradizione si chiamano "rapporti di lavoro" - in cui l'applicazione di nuove 
tecnologie rischia di rendere obsolete o insufficienti le tradizionali regole, che devono essere adattate alle esigenze di governo di questi nuovi rapporti. Si pensi, per fare solo qualche esempio, da un lato alle nuove forme di lavoro svolto "da remoto" o che si avvalgono di mezzi informatici come le "piattaforme", alle forme di commercio elettronico, all'assetto regolativo di servizi pubblici come i taxi nelle città. Sono altrettante sfide per i "legislatori", che cercano di adeguare e rendere efficienti, ai fini sociali ritenuti meritevoli, le regole dei diversi settori.

\section{INTERNET E LA “DELOCALIZZAZIONE” DEL DIRITTO}

L'avvento e lo sviluppo delle tecniche informatiche pongono problemi nuovi ai legislatori e agli applicatori del diritto. Si pensi al tema della "localizzazione" della fonte e della diffusione dei messaggi, in un mondo dove Internet consente di far partire un messaggio da qualunque luogo, anche non dichiarato, di farlo giungere dovunque a tutti coloro che sono in qualche modo "collegati", e di renderlo permanentemente accessibile a tutti a tempo indeterminato.

Tradizionalmente il diritto tende e regolare fenomeni localizzati e localizzabili, che avvengono all'interno della comunità di riferimento del singolo ordinamento giuridico. Nel mondo moderno una grande parte del diritto è diritto "statale", cioè deciso, imposto e valevole per le persone e i fenomeni che stanno all'interno di un determinato territorio (la "legge territoriale"), in quanto posto e applicato tendenzialmente dagli apparati dello Stato costituito su quel territorio. Tradizionalmente si definiva lo Stato con la triade "un popolo, un territorio, un governo" (cioè un apparato autoritativo). Ebbene, un fenomeno come l'uso, attivo e passivo, di Internet tende a sfuggire, per sua natura, alla localizzazione: in un luogo può trovarsi l'apparato tecnico che serve per collegarsi alla rete, in un altro (anche lontanissimo) il mittente di un messaggio, in un altro ancora il destinatario reale o potenziale.

Di qui la difficoltà per il diritto di porre regole valide ed efficaci per i fruitori, e anche di scegliere la "legge" applicabile. Il tradizionale "diritto internazionale privato" - che in realtà è diritto interno di ogni Stato - stabilisce le regole per determinare la legge "applicabile" ad un determinato rapporto in relazione alla localizzazione delle persone o delle cose che ne sono soggetti o oggetto. Ma qual è la legge applicabile ad un rapporto che si costituisce e si svolge sulla base di quelle molte- 
plici diverse localizzazioni? E comunque, la difficoltà di regolare questi aspetti comporta di necessità anche una "delocalizzazione" delle regole giuridiche, e dunque anche modi di produzione del diritto non più legati solo alle singole comunità e ai singoli apparati autoritativi: insomma un diritto "internazionale" o globale, che però non riguarda più solo - come il diritto internazionale classico, almeno fino alla seconda guerra mondiale - i rapporti fra gli Stati, bensì rapporti che coinvolgono soggetti individuali e apparati collocati in diversi territori.

Il che peraltro implica che vengano create e riconosciute regole che riguardano potenzialmente o effettivamente non questa o quella più o meno ristretta comunità umana, ma tutti gli esseri umani viventi nel mondo: come del resto, per altro verso, è già accaduto e sta accadendo col diffondersi delle idee e delle pratiche dei diritti umani universali, e dei relativi apparati normativi e giudiziari (convenzioni sui diritti umani e relative Corti di garanzia). Non è difficile immaginare che dovranno sempre più svilupparsi in futuro regole di questo genere, di portata planetaria, anche per quanto riguarda l'uso delle tecnologie informatiche, che d'altra parte vanno a interferire non di rado proprio col tema dei diritti umani universali e della loro garanzia.

\section{LE “TECNICHE” DELLA VITA UMANA}

Ci sono poi aspetti delle nuove tecnologie che pongono in discussione o rischiano di alterare le stesse basi biologiche della vita umana, e quindi gli aspetti più intimi dell'esistenza. La persona umana, nella nostra accezione del diritto, è il soggetto, attivo o passivo, delle regole giuridiche, in quanto autore o destinatario delle stesse. Le tecniche di manipolazione genetica e la biotecnologia sembrano talora proporsi come possibili strumenti atti a fare della persona un "oggetto", artificialmente modificato o addirittura creato.

Si pensi alla procreazione e ai rapporti, configurati dal diritto, di genitorialità e di filiazione. Da sempre il diritto conosce forme e regole per disciplinare rapporti di filiazione "adottiva", in cui cioè le funzioni, le regole, i diritti e gli obblighi propri del rapporto fra genitori e figli si applicano anche a realtà umane in cui non sono presenti le genitorialità genetica e biologica: per volontà ed effetto del diritto, si supplisce, nei confronti del nuovo nato, alle funzioni della genitorialità, che a causa di eventi e di comportamenti non può fondarsi sulla procreazione "natu- 
rale". Ma oggi, in virtù delle "tecniche" della procreazione "assistita", questa scissione sembra dilatarsi a dismisura, non più come "rimedio" giuridico e sociale ad una situazione involontariamente creatasi, ma come volontaria scissione fra paternità genetica e paternità legale, fra maternità genetica, maternità biologica (la gestazione) e maternità legale. Ed ecco un'altra sfida per il diritto, che deve individuare i fini sociali desiderabili in questo campo e adottare le regole più adatte (controllando, limitando, vietando) per governare l'impiego di queste tecnologie: fino alla frontiera estrema della possibile "produzione" di nuovi esseri umani per via di manipolazione genetica (la clonazione di esseri umani).

\section{IL DIRITTO E L'“INTELLIGENZA ARTIFICIALE”}

Per altro verso, lo sviluppo e l'impiego della "intelligenza artificiale" pone nuovi problemi al diritto: nella misura in cui si prefigura la possibilità che "macchine" prodotte dall'uomo con le sue tecnologie quindi oggetti - vengano programmate e prodotte con autonome funzioni, a loro volta, di programmazione, di "decisione" e di impulso rispetto ad altre "macchine", non più controllabili da parte dell'originario produttore umano. Si rischia così di perdere il controllo "tecnico" di trasformazioni della realtà in tal modo determinate, e di mettere in questione lo stesso concetto giuridico di "responsabilità", in una sorta di catena incontrollabile di "colpe" di "padri" tecnologici che si riversano sui "figli" di queste tecnologie, e viceversa.

Alla fine, comunque, e tornando al punto di partenza, ciò che si deve ribadire, dal punto di vista del diritto come "tecnica (non tecnica) sociale", è che esso deve mantenere la propria funzione di scelta dei fini sociali e di adeguamento delle regole a ciò che serve a questi fini, nel rispetto dell'essenza dell'uomo, quindi anche "governando" da questo punto di vista l'uso della tecnologia (imponendo limiti e condizioni, disciplinando e vietando), senza perdere mai il "timone".

La caratteristica fondamentale e più preziosa dell'uomo è la sua libertà, anche come fonte della sua responsabilità sociale. Salvaguardare la libertà e la dignità dell'essere umano, di ogni essere umano, è l'imperativo categorico irrinunciabile: un imperativo assoluto, anche di fronte a qualsiasi nuova possibilità tecnologica. Come, per fare un paragone, la civiltà giuridica impone di vietare la tortura, anche se in ipotesi impiegata per fini socialmente desiderabili, allo stesso modo 
il diritto deve mantenere ferma, e progressivamente adeguare, la sua funzione di limite e di controllo rispetto all'impiego di qualsiasi nuova possibilità tecnologica là dove esso realizzi o rischi di realizzare una compromissione dell'essenza dell'uomo. 